\title{
Breed-specific pro-inflammatory cytokine production as a predisposing factor for susceptibility to sepsis in the dog
}

\author{
Jean A. Nemzek, DVM, MS, DACVS, Marta D. Agrodnia, DVM, DACVS and Joe G. Hauptman,
} DVM, MS, DACVS

\begin{abstract}
Objective: To determine whether 2 dog breeds with a high risk for parvoviral enteritis, a disease associated with sepsis, produce stronger pro-inflammatory cytokine responses to a stimulus than dogs with a lower risk. Design: Blinded comparison.

Setting: University outpatient clinic.

Animals: Healthy, unrelated, purebred Doberman Pinschers $(n=10)$ and Rottweilers $(n=9)$ with agematched mixed-breed dogs $(n=7)$.

Interventions: Heparinized, whole-blood samples were collected from each dog and incubated for 6 hours with lipopolysaccharide. Plasma was collected, and bioassays were used to determine the concentrations of TNF- $\alpha$ and IL-6. The mean values obtained from the high-risk breeds were compared with the mean obtained from the mixed-breeds.

Measurements and main results: The mean TNF- $\alpha$ production from dogs with a high risk for parvoviral enteritis $(1321 \pm 161 \mathrm{pg} / \mathrm{mL}$; Doberman Pinscher and Rottweiler) was greater $(P<0.05)$ than that from lower risk, mixed-breed dogs $(674 \pm 186 \mathrm{pg} / \mathrm{mL})$. There were no differences in TNF- $\alpha$ levels between Doberman $(1128 \pm 247 \mathrm{pg} / \mathrm{mL})$ and Rottweiler $(1563 \pm \mathrm{pg} / \mathrm{mL})$ breeds or between any breeds with regard to IL-6 production.

Conclusions: The magnitude of TNF- $\alpha$ production by peripheral blood monocytes was the greatest in the dogs with breed-related risk for parvoviral enteritis. However, additional studies are needed to prove a causal relationship between high TNF and predilection for parvoviral enteritis. Regardless, breed appears to be a predisposing factor for variations in cytokine production that could impact the host response to infection and other inflammatory insults.
\end{abstract}

(J Vet Emerg Crit Care 2007; 17(4): 368-372) doi: 10.1111/j.1476-4431.2006.00215.x

Keywords: cytokines, immunology, inflammation

\section{Introduction}

Sepsis is defined as a systemic inflammatory response to infection. ${ }^{1}$ During a systemic response to infection, TNF- $\alpha$ is an early mediator of the pro-inflammatory cascade that yields other cytokines including IL-6 and, eventually, the acute phase proteins. ${ }^{2}$ This response then regulates cellular immune functions and, under

Unit for Laboratory Animal Medicine, University of Michigan, Ann Arbor, MI (Nemzek), Animal Cancer and Imaging Center, Canton, MI (Agrodnia), and the Department of Small Animal Clinical Sciences, College of Veterinary Medicine, Michigan State University, East Lansing, MI (Hauptman).

Address correspondence and reprint requests to:

Jean A. Nemzek, ULAM, University of Michigan, 018 ARF, 1150 W. Medical Center Drive, Ann Arbor, MI 48109.

E-mail: jnemzek@umich.edu optimum conditions, benefits the host through resolution of the infection. However, aberrant systemic inflammatory responses, in particular overly exuberant reactions, may be deleterious to the host. In humans and laboratory animals, studies have shown that increased levels of pro-inflammatory cytokines correlate with the severity of the septic insult and mortality. ${ }^{3-5}$ In clinical veterinary medicine, increasing TNF activity is predictive of mortality in naturally occurring canine parvoviral infection. ${ }^{6}$

A number of intrinsic factors may regulate the intensity of the systemic inflammatory response. In humans and laboratory animals, these factors include concurrent disease, age, and sex. ${ }^{1,7-10}$ In addition, experimental studies have demonstrated that genetic background 
can influence the severity of pro-inflammatory immune responses including TNF- $\alpha$ production and the susceptibility to infection. ${ }^{11,12}$ In septic human patients, the identification of predisposing factors and the potential response to infection have become important for the prediction of response to therapy and outcome. ${ }^{1}$ However, little is known about the factors affecting the systemic inflammatory response and susceptibility to sepsis in the dog.

Given that genetic background may play a role in immune responses in other species, breed may impact the systemic inflammatory response in dogs. Therefore, in this study, endotoxin-induced production of TNF- $\alpha$ and a downstream pro-inflammatory cytokine, IL-6, in whole-blood samples from Doberman Pinschers, Rottweilers, and mixed-breed dogs were investigated. The selection of breeds was based on the significantly increased risk of parvoviral enteritis in Doberman Pinschers and Rottweilers compared with mixed-breed dogs. ${ }^{13-15}$ During the pathogenesis of parvoviral enteritis, the disruption of the intestinal barrier is a potential source for endotoxemia, bacteremia, and subsequent induction of a systemic inflammatory response, particularly TNF- $\alpha$ production. ${ }^{6,16}$ Therefore, it was hypothesized that the magnitude of the pro-inflammatory cytokine response to endotoxin would be greater in dogs with breed-related risk for the development of canine parvoviral enteritis, a disease associated with sepsis.

\section{Materials and Methods}

\section{Study design and animals}

Dogs presented to the university general medicine service for routine physicals and vaccinations were considered for the study. After written consent, purebred adult Doberman Pinschers, Rottweilers, and mixedbreed dogs were included in the study if they met the following criteria: an unremarkable history; normal physical examination; and no familial relationship with other subjects.

A total of $7 \mathrm{~mL}$ of blood was drawn from each dog. Two milliliters was immediately placed in a tube with EDTA for a complete blood count. ${ }^{\text {a }}$ The remaining $5 \mathrm{~mL}$ of blood was mixed with $100 \mu \mathrm{L}$ of heparin (1000 USP units $/ \mathrm{mL})^{\mathrm{b}}$ for the whole-blood assay.

\section{Whole-blood assay}

The whole-blood assay was performed as described elsewhere. ${ }^{17,18}$ Briefly, the heparin anti-coagulated blood from each dog was divided into $1 \mathrm{~mL}$ aliquots in $1.5 \mathrm{cc}$ Eppendorf tubes. One tube of blood served as a control and was immediately centrifuged at $600 \times g$ for 5 minutes. The plasma was collected and stored at
$-20{ }^{\circ} \mathrm{C}$ for later analysis. Lipopolysaccharide (LPS; $10 \mathrm{ng}$, Escherichia coli O111:B4) ${ }^{\mathrm{c}}$ was added to the second tube of blood, and an equal volume of Roswell Park Memorial Institute media (RPMI) ${ }^{\mathrm{d}}$ was added to the third tube of blood. These tubes were placed on a rotator at $37{ }^{\circ} \mathrm{C}$ for 6 hours. The tubes were then centrifuged, and the plasma was collected as described for the control tube.

\section{Cytokine bioassays}

Bioassays were used to detect biologically active cytokines in this study. These assays have been used to detect canine TNF- $\alpha$ and IL- $6 .{ }^{19}$

\section{TNF measurement}

TNF was measured using the WEHI 164 subclone 13 fibrosarcoma cell line bioassay. ${ }^{20}$ In a flat-bottomed, 96well microtiter plate, ${ }^{\mathrm{e}}$ a standard curve of recombinant human $\mathrm{TNF}^{\mathrm{f}}$ and samples were serially diluted in RPMI with $1 \%$ fetal calf serum $(\mathrm{FCS})^{\mathrm{g}}$ and $1 \mathrm{~mm}$ L-glutamine. ${ }^{\mathrm{g}}$ WEHI cells were suspended at a concentration of $5 \times 10^{5}$ cells $/ \mathrm{mL}$ in RPMI 1640 containing $10 \%$ FCS, $1 \mathrm{~mm}$ L-glutamine, $30 \mu \mathrm{g} / \mathrm{mL}$ gentamicin, and $0.5 \mu \mathrm{g} /$ $\mathrm{mL}$ actinomycin $\mathrm{D} . \mathrm{h}$ The suspension was added to the plate $(100 \mu \mathrm{L} /$ well $)$ and incubated overnight in a humidified incubator $\left(37^{\circ} \mathrm{C}\right.$ and $\left.5 \% \mathrm{CO}_{2}\right)$. At least 18 hours later, Cell Proliferation Reagent WST- $1^{\mathrm{i}}$ was added to the plates $(10 \mu \mathrm{L} /$ well). The absorbance was read 24 hours later on a biokinetics plate reader at $465 \mathrm{~nm}$ with a reference wavelength at $630 \mathrm{~nm}$. The results were calculated using standard software. ${ }^{j}$

\section{IL-6 measurement}

IL-6 was measured with a B-9 cell line bioassay following previously described methods. ${ }^{21}$ In a 96-well microtiter plate, ${ }^{\mathrm{e}}$ a standard curve of human recombinant IL- $6^{\mathrm{k}}$ and samples were serially diluted in Iscove's modified Dulbecco's medium ${ }^{\mathrm{g}}$ containing 5\% FCS, $1 \mathrm{mM}$ L-glutamine, ${ }^{\mathrm{g}} 100 \mathrm{U} / \mathrm{mL}$ penicillin, and $100 \mathrm{U} /$ mL streptomycin. ${ }^{\mathrm{d}}$ The $\mathrm{B} 9$ cells were washed twice and then re-suspended at a concentration of $5 \times 10^{5}$ cells/ $\mathrm{mL}$ in the same media supplemented with $0.05 \mathrm{~mm} 2-$ mercaptoethanol. ${ }^{c}$ The cells were added to the plate $(100 \mu \mathrm{L} /$ well), and the plates were incubated for 3 days in a humidified chamber $\left(37^{\circ} \mathrm{C}\right.$ and $\left.5 \% \mathrm{CO}_{2}\right)$. Cell proliferation reagent WST- 1 was then added to the plates (10 $\mu \mathrm{L} /$ well). The absorbance was read 7 and 24 hours later on a biokinetics plate reader at $465 \mathrm{~nm}$ with a reference wavelength at $630 \mathrm{~nm}$.

\section{Statistics}

Age, total white blood cell (WBC) count, monocyte count, and plasma levels of TNF- $\alpha$ and IL- 6 were compared among the 3 breeds (Doberman, Rottweiler, 
mixed-breed) by one-way ANOVAs. Post-hoc comparisons were made of the orthogonal contrasts of: (1) Doberman and Rottweiler (high-risk breeds) versus mixed and (2) Doberman versus Rottweiler. Data were presented as the mean \pm standard error of the mean. A $P$ value of $<0.05$ was considered significant.

\section{Results}

A total of 26 dogs were entered in the study (Doberman Pinscher $n=10$, Rottweiler $n=9$, mixed-breed $n=7$ ). All subjects were healthy according to their histories and physical examinations. There was no significant difference in the mean age of the Doberman Pinscher, Rottweiler, or mixed-breed groups (5.6 $\pm 0.9,6.5 \pm 0.9$, and $4.8 \pm 1.3$ years, respectively). The gender distribution was as follows: Doberman Pinscher, 9 females (8 spayed, 1 intact) and 1 neutered male; Rottweiler, 3 spayed females and 6 males ( 4 neutered and 2 intact); and mixed-breed, 2 spayed females and 5 neutered males.

There were no significant differences in the mean total WBC counts of the Doberman Pinscher, Rottweiler, and mixed-breed groups $(10.5 \pm 0.71,10.9 \pm 1.0$, and $9.0 \pm 0.9 \times 10^{3}$ cells $/ \mu \mathrm{L}$, respectively). Likewise, the monocyte counts of Doberman Pinscher, Rottweiler, and mixed-breed groups did not differ significantly $\left(0.42 \pm 0.05,0.53 \pm 0.12\right.$, and $0.40 \pm 0.05 \times 10^{3}$ cells $/ \mu \mathrm{L}$, respectively).

Bioassays for TNF- $\alpha$ and IL- 6 were run on the 3 plasma samples obtained from each dog. The control, non-incubated samples showed no detectable levels of either TNF- $\alpha$ (assay lower limit of detection $=150 \mathrm{pg}$ / $\mathrm{mL}$ ) or IL-6 (assay lower limit of detection $=14 \mathrm{pg} / \mathrm{mL}$ ) in any of the dogs. Likewise, none of the incubated control samples contained detectable levels of the proinflammatory cytokines. These findings confirmed that there was no recent in vivo stimulation of systemic inflammation in the study subjects and that incubation alone did not induce cytokine production. In samples stimulated with LPS and incubated for 6 hours, the proinflammatory cytokines were readily detectable. The TNF- $\alpha$ and IL- 6 levels obtained from the whole blood of $1 \mathrm{dog}$ in the Rottweiler group were greater than the mean $\pm 30 \mathrm{SD}$ and mean \pm 4 SD (respectively) of the remaining dogs in that group. Therefore, this dog was considered an outlier and removed from the study. The mean TNF- $\alpha$ concentration produced in the in vitro system was greater $(P<0.05)$ in the group considered to be at high risk for parvoviral enteritis (Doberman Pinscher and Rottweiler, $1321 \pm 161 \mathrm{pg} / \mathrm{mL}$ ) as compared with the mixed-breed group $(674 \pm 186 \mathrm{pg} / \mathrm{mL})$ (Figure 1). The difference in TNF- $\alpha$ concentration between the Doberman $(1128 \pm 247 \mathrm{pg} / \mathrm{mL})$ and

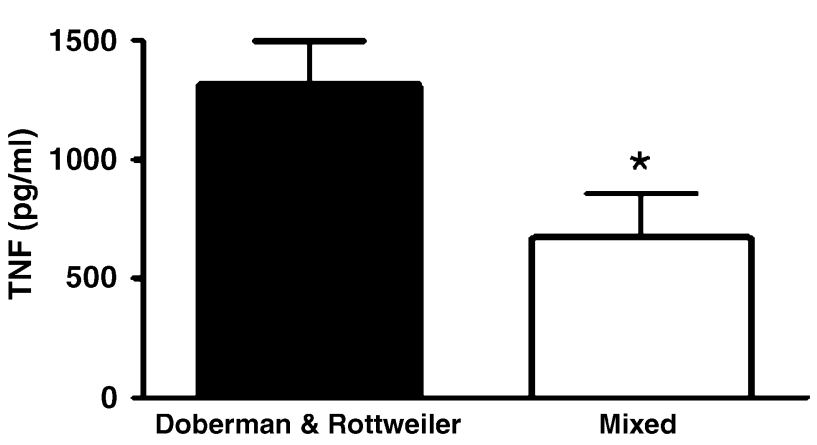

Figure 1: TNF- $\alpha$ production in whole blood. Whole-blood samples from Doberman Pinscher $(n=10)$ and Rottweiler $(n=9)$ dogs were stimulated with lipopolysaccharide for 6 hours. TNF$\alpha$ was measured in plasma via WEHI bioassay. Compared with similarly treated samples from mixed-breed dogs $(n=7)$, TNF- $\alpha$ concentrations were significantly increased. Results are expressed as mean \pm standard error of the mean. $* P<0.05$.

Rottweiler $(1563 \pm 240 \mathrm{pg} / \mathrm{mL})$ groups was not significant $(P>0.05)$. There was no difference in IL-6 production in whole-blood samples between the Doberman Pinscher $(16,543 \pm 2557 \mathrm{pg} / \mathrm{mL})$, Rottweiler $(24,076 \pm 1742 \mathrm{pg} / \mathrm{mL})$, and mixed-breed groups $(23,178 \pm 5224 \mathrm{pg} / \mathrm{mL})$ (Figure 2).

\section{Discussion}

To examine the inflammatory response to LPS for different breeds of dogs, a whole-blood assay was used. Whole-blood assays similar to the one used in this study have been used to study cytokine responses in a number of species including humans, wildlife, and rodents. ${ }^{17,18,22}$ Essentially, this assay assesses the cytokine production of peripheral mononuclear cells. Although the function of peripheral mononuclear cells may not

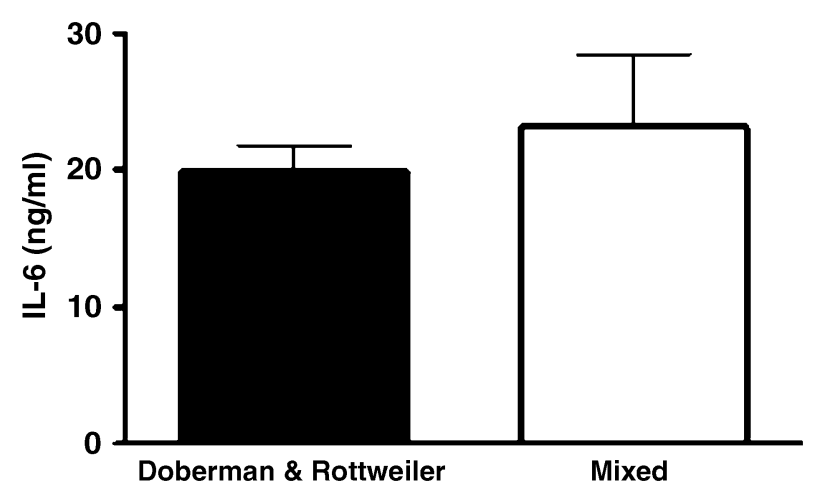

Figure 2: IL-6 production in whole blood. Whole-blood samples from Doberman Pinscher $(n=10)$, Rottweiler $(n=9)$, and mixed-breed dogs $(n=7)$ were stimulated with lipopolysaccharide for 6 hours. IL- 6 was measured in plasma via B-9 cell bioassay. Results are expressed as mean \pm standard error of the mean. 
always be representative of tissue-fixed macrophage populations, ${ }^{7}$ the whole-blood assay does have a number of advantages. Most notably, the assay allows the study of cytokine production in clinical patients without risk to the individuals. The cells may then be studied in an environment that is more representative of the in vivo situation than with cell separation and culture. In addition, identical samples of whole blood may be exposed to a number of different stimuli to examine the contribution of various cellular pathways in the inflammatory response. In this case, endotoxin is considered to play a role in the pathogenesis of parvoviral disease; therefore, LPS was chosen as the stimulus for the whole blood. It is important to note that the mononuclear cell counts of the blood samples drawn from the different breeds of dogs were not significantly different, establishing that any detected differences in cytokine levels would have been the result of differences in mononuclear cell function.

Using the whole-blood assay, an attempt was made to determine whether cytokine production in response to endotoxin would vary among breeds of dogs. In this study, the mean TNF- $\alpha$ production was greater in whole blood from the Rottweilers and Dobermans than mixed-breed dogs. However, IL-6 production was not different among the groups. This finding could simply be due to the sample timing. IL- 6 production generally lags behind TNF- $\alpha$ production and therefore the peak values may not have been achieved at the time the samples were harvested. These findings illustrate 2 interesting points. First, pro-inflammatory cytokine production, specifically TNF- $\alpha$, may vary among breeds. Second, this breed-related variation in cytokine production may be associated with predilection to a known cause of sepsis, parvoviral enteritis. Of course, the connection between TNF- $\alpha$ and parvoviral enteritis is speculative and little is known about the responses of these breeds to other inflammatory conditions. However, the effect of genetics on the inflammatory response to infection has been documented in other species. An experimental study demonstrated that the production of TNF- $\alpha$ and IL- 6 will influence the manifestation of pneumonia in mice exposed to Myobacterium pulmonis and is dependent upon the genetic background of the test subjects. ${ }^{11}$ Strain also played a role in the TNF- $\alpha$ responses to trauma and hemorrhage in a mouse model. ${ }^{12}$ Therefore, the results of this study suggest that further evaluation of breed as a pre-morbid factor affecting canine sepsis is warranted.

The contribution of breed predisposition to TNF- $\alpha$ and IL-6 production cannot be completely defined until several concurrent factors have been evaluated. Sex hormones modulate inflammatory responses in murine models of trauma, followed by hemorrhage and sep- sis. ${ }^{7,8}$ Estradiol appears to have beneficial effects and reduces mortality in these conditions. Similarly, with naturally occurring parvoviral enteritis in dogs over 6 months of age, intact males are twice as likely as females to develop disease. ${ }^{14}$ In the present study, spayed females were over-represented in the Doberman Pinscher group in comparison with the other 2 groups, and the impact of this difference must be considered. However, recent trauma and sepsis experiments indicate that, unlike tissue macrophages, peripheral monocytes may not demonstrate sex-related differences of TNF- $\alpha$ and IL-6 in in vitro systems. ${ }^{7}$ Those studies and the fact that the majority of subjects in this study were spayed or neutered suggest that sex hormones were not a factor in determining the cytokine responses. In addition, age must be considered when evaluating inflammatory responses. Experimental studies have shown that TNF- $\alpha$ and IL-6 production in response to inflammatory insults will increase with age. ${ }^{9,10}$ As there was no significant difference in age between the breed groups in this study, age did not contribute to the differences in cytokine levels.

Although the evaluation of predisposing and premorbid factors appears to be quite complex, this consideration is an important step in understanding sepsis. In human medicine, the intricacies of diagnosing and treating sepsis prompted the development of the PIRO staging system. ${ }^{1}$ This system classifies patients according to predisposing conditions, infection, response of the host, and organ dysfunction. The patient population can then be stratified to define complex host-pathogen interactions that impact treatment and eventual outcome. Although initially daunting, a similar approach in clinical veterinary medicine would provide a wealth of information concerning sepsis in the dog.

In summary, this study used a non-invasive wholeblood assay to assess the pro-inflammatory cytokine response to endotoxin in hospital populations of adult Doberman Pinscher, Rottweiler, and mixed-breed dogs. The results demonstrated no differences in peripheral cell counts or IL-6 production but did show increases in TNF- $\alpha$ production in the purebred dogs, particularly in the Rottweilers. Although the increased TNF- $\alpha$ production was documented in 2 dog breeds with a known predilection for parvoviral enteritis, it is important to note that the cytokine production and development of disease cannot be directly linked at this time. Further studies are needed to evaluate factors affecting the systemic inflammatory response and susceptibility to sepsis in the dog. Of specific interest is how genetic background (breed) may influence the severity of the pro-inflammatory immune response, including TNF- $\alpha$ production, following exposure to and infection with canine pathogens such as canine parvovirus. Continued 
evaluation of predisposing factors and the host response to infection in the dog will provide an important insight into the complex diagnosis and treatment of sepsis.

\section{Acknowledgements}

Funding: These studies were supported by Companion Animal Fund, College of Veterinary Medicine, Michigan State University, East Lansing, MI.

\section{Footnotes}

a Advia 120, Bayer Healthcare LLC, Tarrytown, NY.

b Baxter Healthcare, Deerfield, IL.

Sigma, St. Louis, MO.

d Bio-Whittaker, Walkersville, MD.

e Costar, Cambridge, MA.

f Cetus Corp., Emeryville, CA.

g Gibco, Grand Island, NY.

h Calbiochem, La Jolla, CA.

i Boehringer-Mannheim, Mannheim, Germany.

KC4, Kineticalc, Bio-Tek Instruments Inc.

k Pepro-Tech, Rocky Hill, NJ.

\section{References}

1. Levy M, Fink M, Marshall JC, et al. 2001 SCCM/ESICM/ACCP/ ATS/SIS international sepsis definitions conference. Intens Care Med 2003; 29:530-538.

2. Dinarello CA. Pro-inflammatory and anti-inflammatory cytokines as mediators in the pathogenesis of septic shock. Chest 1997; 12:321S-329S.

3. Ebong S, Call D, Nemzek JA, et al. Immunopathologic alterations in murine sepsis models of increasing lethality. Infect Immunol 1999; 67(12):6603-6610.

4. Remick D, Bolgos GL, Siddiqui J, et al. Six at six: interleukin-6 measured 6 hours after the initiation of sepsis predicts mortality over 3 days. Shock 2002; 17(6):463-467.

5. Oberhoffer M, Vogelsang H, Russwurm S, et al. Outcome prediction by traditional and new markers of inflammation in patients with sepsis. Clin Chem Lab Med 1999; 37:363-368.

6. Otto CM, Drobatz KJ, Soter C. Endotoxemia and tumor necrosis factor activity in dogs with naturally occurring parvoviral enteritis. J Vet Intern Med 1997; 11:65-70.

7. Dienstknecht T, Schwacha MG, Kang S, et al. Sex steroidmediated regulation of macrophage/monocyte function in a two-hit model of trauma-hemorrhage and sepsis. Cytokine 2004; 25:110-118.

8. Jarrar D, Wang P, Cioffi WG, et al. The female reproductive cycle is an important variable in the response to trauma-hemorrhage. Am J Physiol Heart Circ Physiol 2000; 279:H1015-H1021.

9. Kang S, Matsutani T, Choudhry MA, et al. Are the immune responses different in middle-aged and young mice following bone fracture, tissue trauma and hemorrhage? Cytokine 2004; 26:223230.

10. Tateda K, Matsumoto T, Miyazaki S, et al. Lipopolysaccharideinduced lethality and cytokine production in aged mice. Infect Immunol 1996; 64:769-774.

11. Faulker JW, Simeeka MK, Davidson JK, et al. Gene expression and production of tumor necrosis factor alpha, interleukin 1, interleukin 6, and gamma interferon in $\mathrm{C} 3 \mathrm{H} / \mathrm{HeN}$ and $\mathrm{C} 57 \mathrm{BL} / 6 \mathrm{~N}$ mice in acute Mycoplasma pulmonis disease. Infect Immunol 1995; 63:4084-4090.

12. Matsutani T, Samy TS, Kang S, et al. Mouse genetic background influences severity of immune responses following trauma-hemorrhage. Cytokine 2005; 30:168-176.

13. Houston DM, Ribble CS, Head LL. Risk factors associated with parvovirus enteritis in dogs: 283 cases (1982-1991). J Am Vet Med Assoc 1996; 208:542-546.

14. Glickman LT, Domanski LM, Patronek GJ, et al. Breed-related risk factors for canine parvovirus enteritis. J Am Vet Med Assoc 1985; 187:589-594.

15. Brunner CJ, Swango LJ. Canine parvovirus infection: effects on the immune system and factors that predispose to severe disease. Compend Contin Educ Pract Vet 1985; 7:979-988.

16. Prittie J. Canine parvoviral enteritis; a review of diagnosis, management and prevention. J Vet Emerg Crit Care 2004; 14:167-176.

17. DeForge LE, Remick DG. Kinetics of TNF, IL-6, and IL-8 gene expression in LPS-stimulated whole blood. Biochem Biophys Res Commun 1991; 174:18-24.

18. Call DR, Jacoby M, Rudolph KM, et al. Detecting wildlife orthologues for tumor necrosis factor (TNF) and interleukin 6 (IL-6). Northwest Sci 2000; 74(4):340-345.

19. Carter SD, Barnes A, Gilmore WH. Canine rheumatoid arthritis and inflammatory cytokines. Vet Immunol Immunopathol 1999; 69:201-214.

20. Eskandari MK, Nguyen DT, Kunkel SL, et al. WEHI 164 subclone 13 assay for TNF; sensitivity, specificity, and reliability. Immunol Invest 1990; 19:69-79.

21. DeForge LE, Takeuchi E, Nguyen DT, et al. Immunological priming attenuates the in vivo pathophysiological response to lipopolysaccharide. Comparison of cytokine production, tissue injury, lethality in complete Freund's adjuvant-primed mice and in unprimed mice. Am J Pathol 1994; 144:599-611.

22. Nemzek JA, Morrison L, Peterson JE, et al. Quantification of TNF and IL-6 bioactivity in response to lipopolysaccharide in the degu (Octodon degus). Contemp Topics Lab Anim Med 2003; 42(1): 30-33. 